\title{
Experiencias recientes para el desarrollo y evaluación de competencias transversales en el ámbito de la Ingeniería Mecánica y Ciencia de los Materiales
}

J. Carballeiraa ${ }^{\text {a1 }}$, J. Giner-Navarro ${ }^{1}$, J. Martínez-Casas ${ }^{1}$, A. Sonseca ${ }^{1}$, J.L. Suñer ${ }^{1}$, P. Vila $^{1}$, A. Pedrosa ${ }^{1}$, O. Sahuquillo ${ }^{1}$, F.D. Denia ${ }^{1}$, J.J. Ródenas ${ }^{1}$, M. Tur ${ }^{1}$, M.J. Rupérez ${ }^{1}$ a(jacarmo@mcm.upv.es)

${ }^{1}$ Departamento de Ingeniería Mecánica y de Materiales

Universitat Politècnica de València, Camino de Vera s/n, 46022, Valencia

\begin{abstract}
This paper presents some activities developed in the last two years from the Innovation and Educational Quality Team of the Universitat Politècnica de València, INTEGRAL, to work and evaluate generic competences in subjects within the field of Mechanical Engineering and Materials Science. The idea is to show examples of learning activities that allow developing these competences in technical-scientific subjects, based on previous experiences of the components of the Team, and drawing conclusions and proposals for improvement after their implementation in recent courses.
\end{abstract}

Keywords: generic competences, learning activities, assessment.

\section{Resumen}

En esta comunicación se presentan las actividades más relevantes desarrolladas en los dos últimos cursos desde el Equipo de Innovación y Calidad Educativa de la Universitat Politècnica de València, INTEGRAL, para trabajar y evaluar competencias transversales en asignaturas del ámbito de la Ingeniería Mecánica y de Ciencia de los Materiales. La idea es ofrecer ejemplos de actividades de aprendizaje que permiten desarrollar estas competencias en asignaturas de carácter técnico-científico, partiendo de experiencias anteriores de los componentes del Equipo, y extrayendo conclusiones y propuestas de mejora tras su implantación en cursos recientes.

Palabras clave: competencias transversales, actividades de aprendizaje, evaluación. 
Experiencias recientes para el desarrollo y evaluación de competencias transversales en el ámbito de la Ingeniería Mecánica y Ciencia de los Materiales

\section{Introducción}

El Equipo de Innovación y Calidad Educativa, INTEGRAL - Equipo de INnovación docente para el desarrollo de actividades y herramientas de evaluación de compeTEncias transversales en InGeniería Mecánica y de MateRiALes, tiene como objetivo principal la generación de actividades y herramientas de evaluación de competencias transversales para poder trabajarlas y medir el nivel de logro de los estudiantes. Inicialmente sobre materias relacionadas con la Ingeniería Mecánica y de Materiales, pero con ánimo de extender los resultados a otras disciplinas a partir de la generalización de los materiales desarrollados.

La presente publicación va en la línea marcada por este objetivo. Se van a mostrar cuatro experiencias recientes impulsadas por profesores del Equipo en asignaturas de diversos grados y másteres de la Universitat Politècnica de València (UPV). En concreto, se van a presentar las experiencias más relevantes llevadas a cabo desde el último INRED en el que se presentó una recopilación similar de trabajos anteriores (Carballeira et al., 2017).

Además de estas actividades desarrolladas en el marco del objetivo principal del Equipo, sus componentes también han participado en otras contribuciones relacionadas (Reyes-Tolosa et al., 2018) y como docentes en el curso del ICE de la UPV para la formación de profesorado sobre "CT Análisis y Resolución de problemas" celebrado en enero de 2018. Del mismo modo, han preparado una solicitud para un Proyecto de Innovación y Mejora Educativa que se llevará a cabo en los cursos 2018-19 y 2019-20, y que ha sido concedido con una valoración muy favorable. Y también han asesorado a otros profesores interesados en iniciarse en la innovación docente.

La idea principal de esta contribución es mostrar diferentes ejemplos de actividades de aprendizaje que se pueden llevar a cabo en asignaturas de marcado carácter científico-técnico para trabajar competencias transversales sin que supongan una alteración excesiva del plan de estudios tradicional. Es decir, se pretende mantener en la medida de lo posible los contenidos específicos de los cursos, pero modificando la metodología para poder incorporar las competencias transversales. Según las experiencias previas del Equipo (Carballeira et al., 2015 y 2016), las actividades se deben planificar de forma que para ser completadas y superadas con éxito los estudiantes deban poner en juego las competencias transversales correspondientes.

La primera experiencia se refiere a una actividad de aprendizaje que se desarrolla en una asignatura del Grado en Ingeniería Eléctrica que trata la Teoría de Máquinas y Mecanismos (Suñer et., 2018). Esta asignatura es común en la mayoría de grados en ingeniería de carácter industrial. La actividad propuesta consiste en el análisis completo de un mecanismo real realizado en equipo. Incluye el modelado del mecanismo y la creación de una maqueta o prototipo real. Se emplea la coevaluación para favorecer el desarrollo de competencias transversales. Participaron 76 estudiantes agrupados en equipos de 4.

La segunda experiencia se lleva a cabo en una asignatura del Máster Universitario en Ingeniería Industrial sobre Tecnología de Máquinas (Pedrosa et al., 2018). Se diseñaron y pusieron en marcha dos actividades para poder evaluar las competencias "Análisis y Resolución de Problemas” y "Diseño y Proyecto”, CT03 y CT05, respectivamente, según la 
clasificación empleada en el proyecto institucional (UPV, 2014). Para ello se emplearon las herramientas Exámenes y Tareas de la plataforma PoliformaT. Hubo 40 estudiantes en esta experiencia.

La tercera experiencia se desarrolla en la asignatura de Mecánica, en el $2^{\circ}$ curso del Grado en Ingeniería Aeroespacial (Giner-Navarro et al., 2019 - critical thinking). Se trata de una actividad para evaluar la competencia en "Pensamiento Crítico" (CT09). En este caso se optó por realizar una charla informativa a los estudiantes acerca de las bondades de una aplicación informática para la resolución de problemas de ingeniería relacionados con la asignatura. Más tarde, se pasó un cuestionario con el que se pretendía medir el grado de desarrollo de su capacidad para el pensamiento crítico, en función de los indicadores facilitados desde el proyecto institucional (UPV, 2014). Se evaluaron 116 estudiantes en esta asignatura.

La cuarta experiencia hace referencia a la evaluación de la competencia transversal CT13, "Instrumental Específica" (UPV, 2014). Se lleva a cabo en dos asignaturas de máster: Ampliación de Vibraciones, correspondiente al primer curso del Máster en Ingeniería Aeronáutica; y Diseño de Máquinas, integrada en el primer año del Máster en Ingeniería Mecatrónica (Giner-Navarro et al., 2019 - instrumental skills). En ambos casos se aprovecharon las sesiones de prácticas para desarrollar esta competencia, de forma que los estudiantes tuvieran dominio sobre las herramientas empleadas en estas sesiones, además de conocer sus ventajas e inconvenientes en comparación con otras similares. Para evaluar la competencia se diseñaron unos cuestionarios específicos. Eran grupos pequeños de unos 15 estudiantes cada uno.

\section{Objetivos}

Los objetivos principales de estas innovaciones han sido:

- Por un lado, la incorporación de nuevas actividades de aprendizaje para el desarrollo de competencias transversales, que se combinen en mayor o menor medida con los contenidos específicos de la asignatura y tengan un peso relativo en la evaluación final de la asignatura, de forma que aumenten la motivación de los estudiantes y la profundidad de su aprendizaje.

- Por otro lado, dar respuesta a la solicitud realizada desde las Estructuras Responsables de los Títulos (ERT), en el marco del proyecto institucional, para la evaluación de las competencias transversales correspondientes a cada asignatura.

La idea era diseñar y planificar actividades que no supusieran un cambio drástico en la metodología docente, ni en los contenidos específicos, dado que muchas de ellas se iban a incorporar con el curso ya iniciado, y además, se pretendía facilitar esta tarea a los profesores más escépticos.

\section{Desarrollo de la innovación}

\subsection{Teoría de Máquinas y Mecanismos, Grado en Ingeniería Eléctrica}


Experiencias recientes para el desarrollo y evaluación de competencias transversales en el ámbito de la Ingeniería Mecánica y Ciencia de los Materiales

La primera experiencia que se va a describir es la propuesta de un trabajo académico en equipo sobre un sistema real relacionado con la temática de la asignatura. Esta metodología no es novedosa, y se propone de forma habitual en muchas asignaturas, pero la forma en que se incorporaron y evaluaron las competencias transversales puede ser un ejemplo de buenas prácticas.

La actividad se llevará a cabo siguiendo los siguientes términos:

1. El trabajo se realiza en grupos de cuatro estudiantes. Todos los miembros del grupo tendrán la misma nota en el trabajo.

2. El grupo de trabajo propone al profesorado el mecanismo sobre el que trabajará. Deberá ser un mecanismo de un sistema real que contenga las características (barras, pares cinemáticos, actuadores, etc.) mostradas en las clases de aula y que permita realizar sobre él los análisis necesarios. El profesorado dará el visto bueno al mecanismo, en un tutoría concertada, teniendo en cuenta que no haya grupos con el mismo mecanismo y que el mecanismo pueda ser analizable al nivel de la asignatura, y dará directrices al grupo para enfocar los diferentes análisis.

3. El grupo realiza un modelo del mecanismo real, que puede ser construido con aquellos componentes y materiales que el grupo estime oportuno, como por ejemplo sistemas de contrucción del ámbito del juguete, componentes de ferretería o incluso pueden construir sus propios componentes en el laboratorio de impresión 3D de la ETSID. También puede realizarse un modelo virtual del mecanismo utilizando programas como Working Model 2D, Solidworks o Autodesk Inventor. O bien ser recuperado de algún desgüace o taller.

4. El grupo realiza una memoria que consta de una introducción, una breve reseña histórica del mecanismo y se describen diferentes alternativas al mecanismo seleccionado para realizar el mismo trabajo; se incluirán los análisis cinemático, dinámico inverso y dinámico directo del mecanismo estudiado, con un formato similar a los problemas realizados en clase. Los datos de los diferentes análisis serán revisados por el profesorado en una tutoría concertada para ello. Además, la memoria incluirá un presupuesto del trabajo realizado, contabilizando horas de trabajo, coste de materiales, etc. La correción de la memoria será realizada por el profesorado y valdrá el $50 \%$ de la nota de la actividad.

5. El grupo realiza una defensa oral del trabajo, en la que intervendrán todos los componentes del grupo y con un orden establecido de manera aleatoria en el momento de iniciar la defensa. La defensa se realiza en presencia del profesorado y de otros grupos de trabajo con una exposición de 10 minutos y un turno de preguntas del profesorado y el resto de grupos de 5 minutos. La evaluación de la defensa oral la realizarán el profesorado, con un peso del $70 \%$ y los grupos presentes, haciendo una coevaluación (3 grupos por sesión; 2 evalúan al otro), con un peso del 30\%. El peso de la defensa oral será del $50 \%$ de la actividad.

6. El peso total de la actividad en el conjunto de la asignatura es del $25 \%$ de la nota de la asignatura. 
Se debe resaltar que para la realización de la corrección de las diferentes partes se cuenta con rúbricas generadas previamente por los diferentes Equipo de Innovación y Calidad Educativa de la UPV (UPV, 2014) y una lista de control creada especificamente para la actividad y que se muestra en la Figura 1. Esta lista de control se utiliza para la evaluación de la exposición oral y es cumplimentada tanto por el profesorado que realiza la heteroevaluación como el alumnado que realiza la coevaluación y tiene, como característica especial, la inclusión de preguntas al grupo evaluador comparando el trabajo propio con respecto al que están evaluando. Para poder puntuar de forma homogénea, se facilita a los estudiantes una guía con orientaciones sobre qué significa cada nota:

\section{3: El análisis está correctamente planteado y estructurado. Los diagramas, métodos} y ecuaciones del análisis están correctamente creados y utilizados.

2: El análisis está bastante bien planteado y estructurado. Algún diagrama, método o ecuación del análisis no está correctamente creado o utilizado.

1: El análisis tiene bastantes deficiencias en el planteamiento y estructura, aunque hay algunos aciertos. Varios diagramas, métodos o ecuaciones del análisis no están correctamente creados o utilizados.

0: El análisis tiene muchas deficiencias, errores e incongruencias.

Teniendo en cuenta que la asignatura "Máquinas y Mecanismos" se imparte en el $2^{\circ}$ curso del GIE, el trabajo de las competencias transversales debe llegar al primero de los tres niveles que el proyecto institucional de la UPV establece para sus estudios de Grado y Máster (UPV, 2014).

Formalmente, sólo se evalúa la competencia transversal CT02 "Aplicación y Pensamiento Práctico”, aunque otras se trabajen de forma implícita (Suñer et., 2018). En este caso, la competencia transversal se puntúa numéricamente con la suma ponderada de la nota del defensa oral (profesor 70\% + coevaluación 30\%) más la nota del informe escrito del apartado 3 de la Figura 1. Esta nota numérica sobre 3 se traduce por tramos a la escala empleada en el proyecto institucional (UPV, 2014): A - Excelente/Ejemplar, B - Bien/Adecuado, C - En desarrollo, D - No alcanzado.

El tiempo invertido para una actividad como esta es un factor importante tanto para el profesorado como para el alumnado. Para el profesorado porque se necesita tiempo para realizar el seguimiento establecido a través de las tutorías y los procedimientos de evaluación, tanto la exposición oral como las memorias escritas. El uso de rúbricas y listas de control facilita esta tarea. Y para el alumnado, porque se necesita tiempo para construir el modelo de mecanismo, realizar los diferentes análisis requeridos y resolver los problemas que surgen de aplicar los conceptos teóricos de clase a sistemas reales. 
Evaluación de la defensa oral del trabajo de Máquinas y Mecanismos del GIE.

Sesión: Turno:

Dia: Hora de inicio:

Grupo que realiza la defensa:

Grupo evaluador:

Todos los apartados se valorarán de 0 a 3 de acuerdo con la guía proporcionada para la evaluación.

\begin{tabular}{|l|l|}
\hline Apartado 1: Realización de la defensa & \\
\hline Apartado 1.1: Exposición oral & \\
\hline Apartado 1.2: Calidad de las diapositivas & \\
\hline Apartado 1.3: Turno de preguntas & \\
\hline Apartado 2: Modelo de mecanismo & \\
\hline Apartado 2.1: Representatividad del modelo & \\
\hline Apartado 2.2: Proceso de creación o recuperación del modelo & \\
\hline Apartado 2.3: Utilidad del modelo & \\
\hline Apartado 3: Análisis realizados & \\
\hline Apartado 3.1: Análisis cinemático & \\
\hline Apartado 3.2: Análisis dinámico de fuerzas & \\
\hline Apartado 3.3: Análisis dinámico de movimiento & \\
\hline Apartado 4: Comparativa con trabajo del equipo evaluador & \\
\hline Apartado 4.1: Posibilidad de mejora del trabajo propio & \\
\hline Apartado 4.2: Nivel del trabajo evaluado respecto del propio & \\
\hline
\end{tabular}

Fig. 1 Lista de control para la evaluación de la presentación oral 


\subsection{Tecnología de Máquinas para la nivelación, Máster Universitario en Ingeniería Industrial}

La motivación de la puesta en marcha de estas actividades reside en la necesidad de evaluar las competencias transversales asignadas a la asignatura por la ERT. Para la primera, CT03 “Análisis y resolución de problemas", además de las pruebas escritas de respuesta abierta, se ha adaptado una actividad ya empleada en cursos anteriores consistente en la resolución de problemas cortos a través de la herramienta Exámenes de PoliformaT. Los alumnos realizan cinco pruebas durante el cuatrimestre; aproximadamente, una cada quincena. Estos ejercicios inciden en aquellos aspectos que se han detectado críticos en la evaluación de los estudiantes. Los contenidos y el nivel de las pruebas están adaptados al tipo de alumnado y a los contenidos de la asignatura que se están impartiendo.

Con el fin de evaluar la segunda competencia CT05 "Diseño y proyecto", se ha propuesto un trabajo en el que los estudiantes, en equipos de dos personas, han de diseñar y calcular un reductor de velocidad de engranajes cilíndricos de dientes helicoidales de una etapa. Se propone realizar tres entregables para conjugar los contenidos de estos con la materia que se está impartiendo en clase. En la primera entrega, a partir de los datos aportados a cada equipo, los alumnos dimensionan los ejes según los criterios de rigidez torsional y fatiga. En la segunda entrega, los estudiantes deben seleccionar los rodamientos adecuados y diseñar el sistema de fijación axial para conseguir un extremo fijo y uno libre en cada uno de los ejes. En la última entrega debe llevarse a cabo el cálculo del ancho del engranaje. La entrega de los trabajos se lleva a cabo a través de la herramienta Tareas de PoliformaT.

Se calculan las puntuaciones numéricas para cada competencia transversal a partir de las notas de las diferentes partes de cada actividad, y después esta nota numérica sobre 10 se traduce por tramos a la escala empleada en el proyecto institucional (UPV, 2014): A Excelente/Ejemplar, B - Bien/Adecuado, C - En desarrollo, D - No alcanzado.

\subsection{Mecánica, Grado en Ingeniería Aeroespacial}

Con esta experiencia se pretendía trabajar y evaluar la competencia en "Pensamiento Crítico". Para el Nivel 1 de desarrollo de la competencia, que corresponde al segundo año de Grado según el proyecto institucional (UPV, 2014), el principal resultado de aprendizaje que define el "Pensamiento Crítico" es:

Muestra de una actitud crítica hacia la realidad, pudiendo analizar y cuestionar información, resultados, conclusiones y otros puntos de vista.

Los indicadores correspondientes son:

- Mostrar una actitud crítica hacia la realidad: preguntarse el porqué de las cosas.

- Profundizar un tema con lógica e imparcialidad, contrastando información en fuentes confiables.

- Diferenciar los hechos de las opiniones, interpretaciones o evaluaciones.

- Prever las consecuencias (implicaciones prácticas) de las decisiones. 
Experiencias recientes para el desarrollo y evaluación de competencias transversales en el ámbito de la Ingeniería Mecánica y Ciencia de los Materiales

Esta competencia está conectada con la capacidad de aplicar procesos lógicos y racionales para analizar las distintas partes de un problema y pensar creativamente para generar soluciones innovadoras. De acuerdo con la definición adoptada en el proyecto institucional de la UPV, se trata de desarrollar un pensamiento crítico interesado en los fundamentos en los que se basan las ideas, acciones y juicios, tanto propios como ajenos. El pensamiento crítico va más allá de las habilidades del análisis lógico, ya que implica cuestionar los supuestos subyacentes en nuestras formas habituales de pensar y actuar y, en base a ese cuestionamiento crítico, estar preparado para pensar y actuar de manera diferente. El pensamiento crítico es el pensamiento de las preguntas: ¿por qué las cosas son así?, ¿por qué las cosas no pueden ser de otra manera?, ¿por qué crees que son así?... En consecuencia, diremos que el estudiante la ha desarrollado en la medida en que se pregunta a sí mismo sobre las cosas y está interesado en los fundamentos de las ideas, acciones, evaluaciones y juicios.

En este sentido, se consideró que las sesiones de prácticas informáticas eran un buen escenario para evaluar esta competencia. A lo largo de estas sesiones, los estudiantes aprendieron a usar ADAMS/View $\mathbb{C}$, un software de simulación dinámica para el diseño e análisis de mecanismos. En cada sesión, los alumnos trabajan en parejas desarrollando diferentes casos aplicados de los que tienen que extraer resultados cinemáticos y dinámicos. Cada pareja resuelve un ejercicio personalizado con datos geométricos e inerciales diferentes al problema de referencia desarrollado y resuelto en el manual de la sesión. Como los resultados solicitados son numéricos, se realiza la corrección automatizada del ejercicio, asumiendo un margen de tolerancia del $5 \%$ con respecto a la solución calculada previamente por el profesor. El promedio de las notas de las siete sesiones determina la evaluación de esta parte de la asignatura.

Para evaluar el desarrollo del pensamiento crítico se van a plantear situaciones a los estudiantes que enfrenten sus expectativas frente a la realidad. Se llevó a cabo una charla por parte de la empresa que distribuye el software, y se grabó para futuros cursos. La idea es que los estudiantes tengan información abundante acerca de las capacidades de dicho software.

Como herramienta de evaluación se planteó un cuestionario con preguntas abiertas. Estas preguntas están diseñadas para estimular el razonamiento crítico, alentando al estudiante a hacer juicios de valor y sacar sus propias conclusiones. Se pretende promover la originalidad de las respuestas más allá del conocimiento técnico adquirido, planteando nuevos casos para que valoren las ventajas y desventajas de implementarlos en el software dinámico. Los estudiantes tuvieron 15 minutos para completar el cuestionario, suficiente para responder con calma a cada una de las preguntas. El cuestionario fue entregado en la última sesión, siguiendo el formato presentado en la Tabla 1.

Los 116 cuestionarios del curso fueron evaluados por un mismo profesor, para mantener la homogeneidad en la medida de los posible, que valoró las respuestas en función de los criterios expresados en la rúbrica UPV para esta competencia y de su propia experiencia. 
Tabla 1. Herramienta de evaluación del Pensamiento Crítico en Mecánica del Grado en Ingeniería Aeroespacial: cuestionario abierto

1. "As the world's most famous and widely used Multibody Dynamics (MBD) software, ADAMS improves engineering efficiency and reduces product development costs by enabling early system-level design validation. Engineers can evaluate and manage the complex interactions between disciplines including motion, structures, actuation, and controls to better optimize product designs for performance, safety, and comfort. Along with extensive analysis capabilities, ADAMS is optimised for large-scale problems, taking advantage of high performance computing environments." ¿Con qué afirmaciones del texto coincides más y con cuáles discrepas? Argumenta tus motivos.

2. Explica cuáles son, en tu opinión, las ventajas e inconvenientes de emplear ADAMS/View $\mathbb{C}$ para resolver un problema de tiro parabólico con rozamiento del aire.

3. ¿Qué parámetros consideras más importantes para llevar a cabo un análisis dinámico correcto? ¿Por qué?

4. ¿Cuáles son, desde tu punto de vista, los factores que pueden hacer que los resultados numéricos se alejen de las medidas reales?

\subsection{Ampliación de Vibraciones, Máster en Ingeniería Aeronáutica; y Diseño de Máquinas, Máster en Ingeniería Mecatrónica}

Con estas experiencias se pretendía trabajar y evaluar la competencia "Instrumental Específica". Esta competencia se refiere al uso de herramientas y tecnologías necesarias para la práctica profesional. Su desarrollo implica que el estudiante podrá identificar las herramientas más adecuadas en cada caso, conocer sus utilidades y poder integrarlas y combinarlas para resolver problemas, llevar a cabo proyectos o experimentos.

El resultado de aprendizaje para esta competencia a nivel de máster se expresa como:

Integra correctamente las herramientas avanzadas del campo profesional.

Este nivel de dominio establece tres indicadores diferentes:

1) Identificación de herramientas avanzadas y su utilidad.

2) Manejo de estas herramientas. 
Experiencias recientes para el desarrollo y evaluación de competencias transversales en el ámbito de la Ingeniería Mecánica y Ciencia de los Materiales

3) Selección y combinación de las herramientas adecuadas para llevar a cabo un proyecto profesional o de investigación.

Las dos asignaturas incluidas en esta experiencia tienen una parte experimental y de uso de software importante en sus sesiones de laboratorio, por lo que sus informes de prácticas están esencialmente relacionados con la competencia instrumental específica. El objetivo principal de las actividades propuestas era estudiar si esta competencia puede evaluarse directamente a través de las notas correspondientes a la parte práctica de la asignatura, o si éstas no proporcionan información separada y específica respecto a sus habilidades asociadas. Para ello, se ha propuesto y diseñado una herramienta sistemática basada en un cuestionario tipo lista de verificación que también incluye preguntas abiertas como herramienta de evaluación. Los diferentes ítems tienen la intención de evaluar los conceptos técnicos adquiridos durante las prácticas de ingeniería, que se dividen en prácticas de informática (usando software especializado) y laboratorio experimental. A modo de ejemplo, en la Tabla 2 se muestra la herramienta de la asignatura Ampliación de Vibraciones.

Tabla 2. Herramienta de evaluación de Ampliación de Vibraciones. Prácticas de informática: cuestionario tipo lista de verificación

\section{I1. Identifica las herramientas avanzadas y su utilidad.}

I1.1 ¿En cuál de los siguientes casos de estudio ves más conveniente el uso de Matlab?

a) Malla de Elementos Finitos de una pieza de ámbito industrial.

b) Integración numérica en el dominio del tiempo de la ecuación del movimiento de un sistema mecánico.

c) Diseño 3D de una pieza de ámbito industrial.

I1.2 ¿Cuál crees que es el software más adecuado para llevar a cabo el mallado de elementos finitos de un sólido?
a) Matlab.
b) Mathematica.
c) Ansys.

I1.3 ¿Para qué caso Matlab es una herramienta flexible y adecuada?

a) Integración numérica.

b) Diseño de procesos de fabricación.

c) Modelado geométrico.

I1.4 ¿Matlab es una herramienta útil para el análisis en frecuencia?

a) No, sólo trabaja en el dominio del tiempo.

b) Sólo para dinámica de baja frecuencia.

c) Sí, es una herramienta muy recomendable para el análisis en frecuencia.

I2. Maneja las herramientas avanzadas. Nota de la práctica.

\section{I3. Selecciona y combina las herramientas adecuadas para realizar un proyecto} profesional o de investigación.

I3.1 ¿Permite Matlab importar geometrías de otros software?

a) Sí, permitiendo el cálculo de las propiedades modales, la respuesta vibracional, etc.

b) Sí, pero sólo para su visualización.

c) No. 
I3.2 Se pretende llevar a cabo un análisis de vibraciones de un ala de un avión. ¿Cómo lo abordarías?

a) Todo en Matlab.

b) Se realiza una malla de Elementos Finitos en Ansys, se importa el modelo geométrico y la malla a Matlab y se procede al cálculo dinámico.

c) Todo en Ansys.

I3.3 ¿En qué caso te interesarías en adquirir una licencia de Matlab para el departamento de tu empresa?

a) En el caso de que el departamento se dedicara al cálculo estructural.

b) En el caso de que el departamento se dedicara al diseño y fabricación.

c) En el caso de que el departamento se dedicara a la simulación dinámica de mecanismos.

I3.4 ¿Cuenta Matlab con un generador de vídeos para representar la respuesta vibracional de un sistema?

a) Sí, como comando directo.

b) No, sólo permite representar las deformadas modales.

c) Sí, se construye el vídeo con distintos frames de la respuesta vibracional.

I3.5 ¿Matlab es un software que permite generar un programa de adquisición de datos?

a) Sí, pero no permite el post-procesado de dichos datos.

b) No.

c) Sí, e incluye paquetes para evitar leakage, aliasing e incluso interfaz gráfica.

\section{Resultados}

\subsection{Teoría de Máquinas y Mecanismos, Grado en Ingeniería Eléctrica}

Aparte de las calificaciones obtenidas por los grupos de trabajo, muchos de los resultados que se pueden obtener de esta actividad son más cualitativos que cuantitativos: los estudiantes mejoran su comprensión de conceptos propios de la asignatura y su motivación.

Aún así es cuantificable la influencia de esta actividad en las calificaciones de la asignatura, como se observa en gráfico de la Figura 2. Las notas de las actividades nuevas, cursos 2016/2017 y 2017/2018, son mejores que las de las actividades que se hacían en los dos cursos anteriores, y eso repercute en una ligera mejora en las notas finales de la asignatura. Pese a haber invertido más tiempo en la realización de las nuevas tareas, el alumnado no se ha visto perjudicado en el rendimiento de las otras partes de la asignatura. 


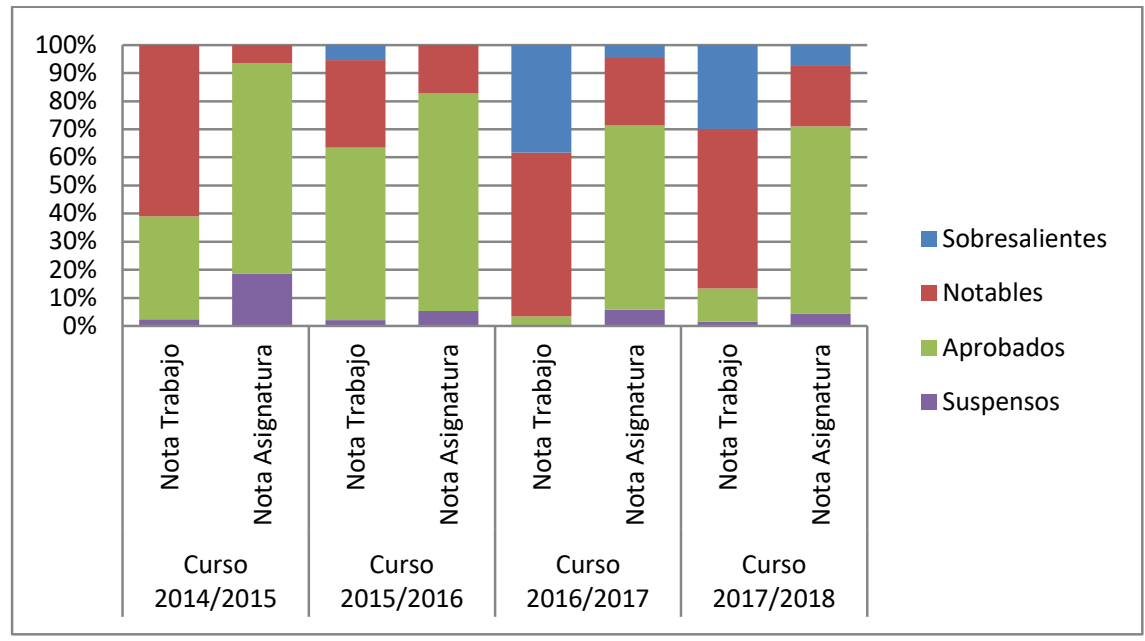

Fig. 2 Calificaciones en los cuatro últimos cursos de trabajos en grupo y final de asignatura, agrupados por niveles de calificación

\subsection{Tecnología de Máquinas para la nivelación, Máster Universitario en Ingeniería Industrial}

Los resultados obtenidos han sido, en general, muy satisfactorios, dado que la aceptación por parte de los estudiantes ha sido buena y han participado en la totalidad de las actividades. Respecto a la actividad relacionada con la CT03, todos han aprobado todos los exámenes PoliformaT y lo que resulta más indicativo, apenas se han detectado fallos en los exámenes en aquellos aspectos trabajados con estas pruebas.

En relación a los trabajos asociados con la competencia CT-05, su aceptación ha sido ligeramente menor ya que se trataba de una actividad menos guiada; sin embargo, los estudiantes la han llevado a cabo correctamente.

Como aspectos a mejorar para el próximo curso se propone, por una parte, el encaje en el calendario de las diferentes entregas del trabajo teniendo en cuenta la carga lectiva de los estudiantes y, por otra, la elaboración de rúbricas para la evaluación.

Los principales indicadores de éxito de las actividades desarrolladas son la excelente tasa de aprobados y la alta correlación que existe entre la evaluación de los trabajos, los exámenes PoliformaT y la nota de la asignatura, como se puede ver en la Figura 3. 


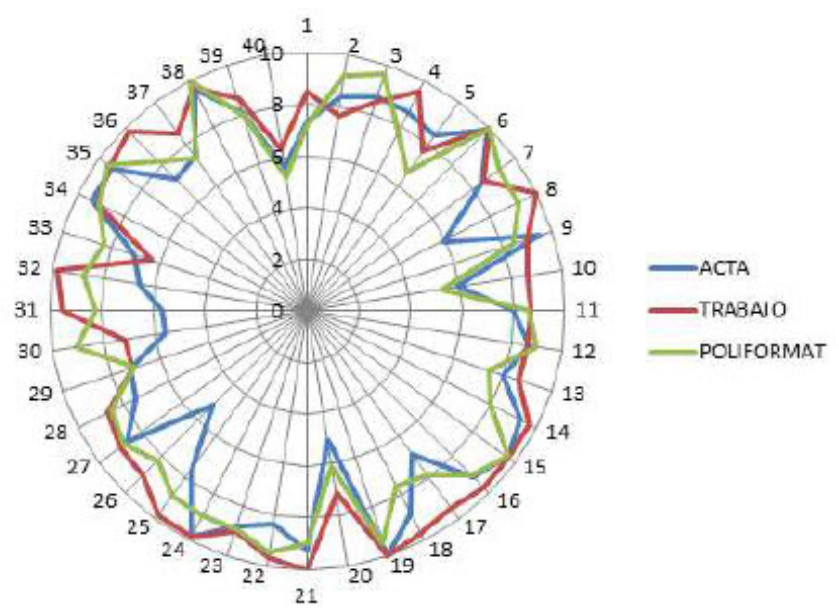

Fig. 3 Correlación de notas obtenidas para cada estudiante

\subsection{Mecánica, Grado en Ingeniería Aeroespacial}

En la Figura 4 se comparan las notas del cuestionario de evaluación de la competencia transversal con la nota de prácticas y la nota del examen de teoría. Un escaso $27 \%$ de estudiantes están fuera del rango de 2 puntos trazado en la Fig. 4(b), que es inferior al 35\% obtenido para la nota de prácticas (Fig. 4(a)). Esta correlación con las notas de teoría permite deducir que el pensamiento crítico es una habilidad importante para abordar la asignatura, aunque no es la única. Se requiere de un proceso lento y estructurado de adquisición e interiorización de conceptos complejos introducidos en las clases de teoría, además de otras competencias transversales como pueden ser las de "Comprensión e integración", “Aplicación y pensamiento práctico" y “Análisis y resolución de problemas” para preparar la asignatura y adquirir las habilidades requeridas en el examen teórico, en el que se plantean problemas dinámicos con una fuerte carga matemática.

(a)

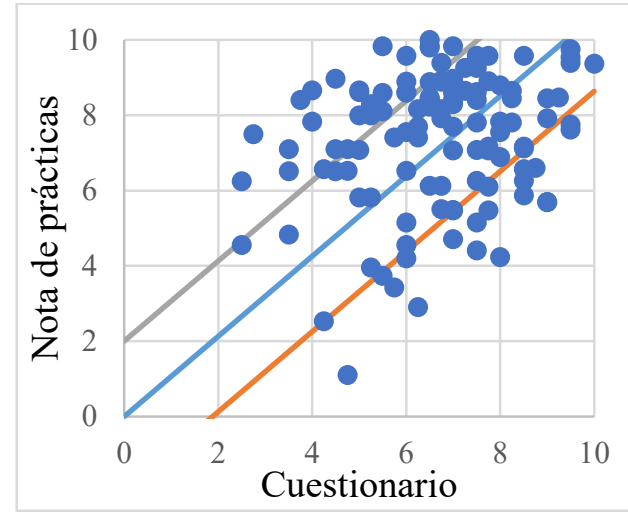

(b)

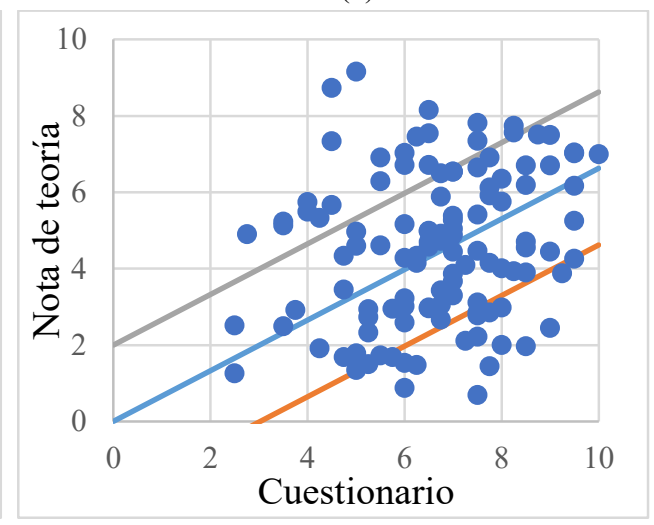

Fig. 4 Correlación notas de la competencia Pensamiento Crítico (Cuestionario) con las notas de prácticas (a) y la de teoria (b) 
Experiencias recientes para el desarrollo y evaluación de competencias transversales en el ámbito de la Ingeniería Mecánica y Ciencia de los Materiales

\subsection{Ampliación de Vibraciones, Máster en Ingeniería Aeronáutica; y Diseño de Máquinas, Máster en Ingeniería Mecatrónica}

Los resultados muestran que existe una cierta correlación entre la competencia transversal y la nota de prácticas de la asignatura. Las habilidades técnicas adquiridas durante las sesiones de laboratorio se muestran de gran interés para preparar los exámenes de acuerdo con la tendencia significativa observada entre la competencia y las notas de teoría y globales. Sin embargo, la correlación observada no es lo suficientemente fuerte como para asignar a la competencia directamente la nota de la asignatura, concluyendo que se requiere una herramienta de evaluación independiente para valorar la competencia instrumental específica.

Las preguntas abiertas han brindado mayor información sobre las habilidades técnicas adquiridas por los estudiantes que la lista de verificación, lo que ha llevado a un replanteamiento del diseño del cuestionario para los próximos cursos.

\section{Conclusiones}

Las principales conclusiones que se pueden extraer de las experiencias llevadas a cabo son:

- Con un buen diseño de la actividad se pueden trabajar varias competencias transversales simultáneamente.

- Se pueden trabajar las competencias sin necesidad de evaluarlas. Las actividades proporcionan al estudiante una base que le ayudará en una nueva situación que las requiera o en las que se vayan a evaluar.

- Para competencias de tipo cognitivo, como el "Pensamiento Crítico", se necesitan metodologías específicas cuidadosamente diseñadas. En opinión de los autores, es difícil inferir una calificación para esta competencia transversal del desempeño en otras actividades de aprendizaje de la asignatura.

- Las preguntas abiertas en los cuestionarios han demostrado ser una herramienta muy potente para la evaluación de competencias transversales ya que, más allá de una respuesta correcta o incorrecta, permiten analizar el razonamiento empleado por el estudiante.

\section{Agradecimientos}

Los autores quieren mostrar su agradecimiento al Vicerrectorado de Estudios, Calidad y Acreditación de la Universitat Politècnica de València por la financiación obtenida a través del programa PIME 2018-19 bajo la referencia DPTO.IMM.

\section{Referencias}

CARBALLEIRA, J., ROVIRA, A., SUÑER, J.L., NADAL, E., RUPÉREZ, M.J., DOLS, J.F., SAHUQUILLO, O., MARTÍNEZ-CASAS, J., VILA, P., PEDROSA, A., DENIA, F.D., RÓDENAS, J.J., TUR, M. (2017). "Diseño de actividades y uso de la coevaluación para fomentar el desarrollo de competencias transversales en ingeniería mecánica y de materiales." en Congreso Nacional de Innovación Educativa y Docencia 
en Red (IN-RED 2017). (ISSN 978-84-9048-568-2). Valencia: Editorial UPV. 940-954. http://dx.doi.org/10.4995/INRED2017.2017.6830

CARBALLEIRA, J., MARTÍNEZ-CASAS, J., SAHUQUILLO, O., SONSECA, A., SUÑER, J.L., VILA, P., DENIA, F. D., RÓDENAS, J.J., MARCO, O. (2016). "Assessment of problem-solving skills and capacity for applying knowledge in practice in subjects related to mechanical and materials engineering" en Advances in Higher Education, Ed. Universitat Politècnia de València, Chapter 5, pp. 71-88.

CARBALLEIRA, J., MARTÍNEZ-CASAS, J., SAHUQUILLO, O., SONSECA, A., DENIA, F.D., SUÑER, J.L., VILA, P., RÓDENAS, J.J., MARCO, O. (2015).

"Desarrollo de estrategias de evaluación de competencias transversales en asignaturas de ingeniería mecánica y de materiales." En Congreso Nacional de Innovación Educativa y Docencia en Red de la Universitat Politècnica de València. (ISSN 978-849048-396-1). Valencia: Editorial UPV. 923-937.

GINER-NAVARRO, J., SONSECA, A., CARBALLEIRA, J., MARTÍNEZ-CASAS, J. (2019) "Assessment of instrumental skills and capacity to use the techniques and tolos in practice within a subject related to mechanical engineering" en 13th annual International Technology, Education and Development Conference (INTED2019). (ISBN 978-84-09-08619-1). Valencia: IATED Digital Libray.

GINER-NAVARRO, J., SONSECA, A., MARTÍNEZ-CASAS, J., CARBALLEIRA, J. (2019) "Assessment of critical thinking within a subject related to mechanical engineering" en 13th annual International Technology, Education and Development Conference (INTED2019). (ISBN 978-84-09-08619-1). Valencia: IATED Digital Libray.

PEDROSA, A., VILA, P. (2018). “Actividades para la evaluación de las competencias CT-03 y CT-05 en la asignatura Tecnología de Máquinas para Nivelación del primer curso del MUII." en JORNADA DE INNOVACIÓN DOCENTE ETSINF 2018 (JIDINF'18). (ISBN 978-84-09-05709-2). Valencia: Editorial Escola Tècnica Superior d'Enginyeria Informàtica.

REYES-TOLOSA, M.D., SAHUQUILLO, O. (2018). "Propuesta de una metodología de evaluación del aprendizaje basada en las TIC." en Congreso Nacional de Innovación Educativa y Docencia en Red (IN-RED 2018). (ISBN 978-84-9048-750-1). Valencia: Editorial UPV. 691-701. http://dx.doi.org/10.4995/INRED2018.2018.8622

SUÑER, J.L., CARBALLEIRA, J. (2018). "Diseño de una actividad para el desarrollo y evaluación de competencias transversales en el ámbito de la Teoría de Máquinas y Mecanismos." en Congreso Universitario de Innovación Educativa en las Enseñanzas Técnicas (26 CUIEET). (ISBN 978-84-17445-03-4). Gijón: Universidad de Oviedo.

UPV (2014). Proyecto institucional sobre Competencias Transversales. http://www.upv.es/contenidos/COMPTRAN/ [Consulta: 25 Marzo 2019] 